\title{
THE PAINFUL SHOULDER
}

\section{Review of One Hundred Personal Cases with Remarks on the Pathology}

\author{
R. J. W. Withers, Belfast, Northern Ireland
}

From the Orthopaedic and Fracture Service, Royal Victoria Hospital, Belfast*

In considering the well-defined group of conditions in which there is limitation or alteration in the range of movement of the shoulder joint, pain on attempted movement, and sometimes muscle wasting, with no radiographic evidence of abnormality, certain anatomical features must be recalled. In the adult the capsule is fused with the overlying short rotator muscles; whereas in infants the capsule is separated from the deep muscles of the shoulder by areolar tissue, the tendons being short and the muscle fibres extending to within a few millimetres of their humeral attachment. With increasing age the tendons become longer and in old age the supraspinatus tendon may extend as far proximally as the suprascapular notch. The degree of adhesion of the tendons to the capsule increases with age, and in older subjects the fusion is complete. The changes are more marked in the upper part of the capsule so that in adult life the entire breadth of the supraspinatus tendon, and the upper parts of the infraspinatus and subscapularis tendons, are intimately fused with the shoulder capsule.

The frequency with which rupture of the supraspinatus tendon is found at post-mortem examination was emphasized by Codman (1931) and Codman and Akerson (1931). Grant and Smith (1948) demonstrated that degenerative changes in the capsule, especially in its upper part, increased in arithmetical progression as age advanced. In ninety-five dissectingroom specimens they found degenerative changes causing complete or incomplete rupture of the capsule in no case of a subject aged 17 to 40 years; in 25 per cent. of subjects aged 40 to 56 years; in 39 per cent. of subjects aged 56 to 76 years; and in 50 per cent. of subjects aged 76 to 86 years. It must also be recognised that falls on the shoulder are more likely to cause injury to the upper part of the capsule than to its anterior or posterior aspects. For these three reasons-fusion of the supraspinatus with the capsule, increasing degeneration of this fused part as years advance, and liability to direct injury from falls-it is evident that most cases of "painful shoulder" are due to pathological changes in the upper part of the capsule.

In this review, a series of one hundred patients have been re-examined at intervals ranging from one to three years after cessation of treatment. The series includes only patients in whom there was no radiographic evidence of abnormality; examples of calcareous deposit in the tendons round the joint have been excluded. The average age was fifty-two years. There were fifty-two men and forty-eight women. The right shoulder was affected in fifty-seven and the left in forty-three patients. There was a definite history of injury in 60 per cent. Three clinical groups were differentiated: 1) rupture of the supraspinatus; 2) supraspinatus tendinitis; 3) capsulitis of the shoulder.

Rupture of the supraspinatus-After a fall on the shoulder there was diffuse pain over the shoulder and sometimes pain referred to the insertion of the deltoid muscle, with inability to elevate the limb, and inability to support it in the elevated position after it had been so placed, but with a normal range of passive movement. In this group the disability was due to loss of the power of fixation of the humeral head to the glenoid by the spinati; the deltoid was thereby put at such a disadvantage that although it was able to contract, as could be felt by the examining hand, it was unable to sustain true shoulder movement. In cases of recent rupture this loss of fixator function may be no more than a reflex phenomenon

* Paper read at the Belfast meeting of the British Orthopaedic Association, October 21, 1948. 
induced by pain, because the uninjured infraspinatus, which is a larger and more important tensor and depressor of the humeral head than the supraspinatus, is also thrown out of action. If this is accepted, it follows that it is impossible on clinical grounds to distinguish between complete and incomplete ruptures. Clinical and operative experience has shown that this is true.

In establishing the diagnosis there is a place for arthrography. Radiographs are taken after the injection of 6 to 8 cubic centimetres of perabrodil ( 35 per cent. solution) either into the shoulder joint as suggested by Oliver Axen (1941) or into the subdeltoid bursa as practised by the writer. It can then be demonstrated in a few minutes whether or not there is communication between the bursa and the joint cavity. It must be recognised, however, that although demonstration of a communication is strongly suggestive of complete supraspinatus tendon rupture it is not absolutely conclusive because in a small percentage of normal joints there is communication with the bursa (Axen 1941).

Supraspinatus tendinitis-In this series there were twenty-seven cases of supraspinatus tendinitis. After direct injury, or sometimes spontaneously, a painful arc of movement during mid-elevation develops, with weakness of the limb and often with abnormal scapulohumeral rhythm but without limitation of the range of movement. Symptoms had been present, on the average, for five months. The disability is often ascribed to impingement of an irritated or inflamed supraspinatus tendon against the under-surface of the acromion, but in two cases in which rupture of the supraspinatus tendon had been treated by suture with excision of the acromion there was still the typically persistent pain of supraspinatus tendinitis. So much bone had been excised that acromial pressure could not have been the cause. The work of Inman, Saunders and Abbott (1944) may throw light on this problem. In myographic studies of the shoulder joint they noted that the peak of contraction of the supraspinatus muscle occurred between 80 and 120 degrees elevation of the limb. This muscle contracts most powerfully in that particular phase of elevation which, in the group under consideration, is associated with pain, and it is possible that active contraction of a supraspinatus tendon which is irritable from inflammation or rupture may in itself be sufficient to cause pain.

Capsulitis-Sixty-one cases were classified as examples of capsulitis occurring spontaneously or after injury. There was pain, limitation of scapulo-humeral movement, weakness and muscle wasting. The term capsulitis is used because it is believed that the essential pathology is inflammation of the capsule from injury or irritating causes within the joint. Neviaser (1945) explored ten shoulders in order to determine the underlying pathology and found that the capsule was thickened and adherent to the humeral head; when the joint was manipulated the capsule separated from the head of the humerus in the same way that an adhesive bandage may be torn from the skin; and microscopic section of the capsule showed inflammatory and reparative changes. If this view is accepted it is clear that two stages of the pathological process may be recognised: 1) an early stage of acute inflammation, with inflammatory exudation in and round the capsule and limitation of shoulder movement due to muscle spasm which relaxes under anaesthesia; 2) a later adhesive stage, after resolution of the inflammatory process, in which there are fibrous adhesions limiting movement even when the muscles are relaxed by anaesthesia. This concept of the pathology is borne out clinically, and it is my routine practice to examine every " frozen shoulder " under anaesthesia in order to test the range of movement and put each case into its proper sub-group of irritative or adhesive capsulitis.

\section{TREATMENT AND RESULTS}

Rupture of the supraspinatus tendon-Of the twelve patients in this group, five were treated conservatively and seven by operation. Conservative treatment consisted in complete rest of the shoulder joint in an abduction splint until the patient was able to raise the limb actively. This took an average period of five weeks. When this stage was reached the splint was

vol. $31 \mathrm{~B}$, No. 3, AUgust 1949 
discarded and gentle exercises were practised, at first in the supine position. Three patients regained full painless movement in twelve weeks; in the other two patients three-quarters of the normal range of movement was restored. All returned to full work. Operative treatment was carried out in seven patients, complete rupture of the tendon being found in every case. The acromion process was excised and the tendon was repaired. Two patients gained full recovery of function; two regained almost full movement but with weakness and pain at mid-elevation; and three were not improved at all. The most successful results were secured by simple longitudinal closure of the hiatus without attempting to suture any part of the tendon to the tuberosity.

Supraspinatus tendinitis-Of the twenty-seven patients in this group, thirteen gained full recovery without any treatment at all, or after simple rest in an abduction splint for five or six weeks. Operative treatment was advised in fourteen patients and carried out in eleven. In each case the acromion process was first excised. When the subdeltoid bursa was opened it was found in eight patients that there was a localised L-shaped or longitudinal tear of the upper part of the capsule; in two there was swelling and longitudinal splitting of the upper surface of the supraspinatus tendon; and in one there was rupture of the articular surface of the tendon discovered only after longitudinal incision by which to explore the "lump" in it. This group of cases illustrates the difficulty in differentiating clinically between simple tendinitis and actual rupture of the supraspinatus. Of the nine patients with incomplete rupture of the tendon the most successful results were gained in those in whom it was possible to bring the margins together longitudinally without tension. They recovered full painless movement within four months with no more than slight residual weakness when tested against resistance. All patients in whom there was a more complete rupture, and an attempt was made to suture the tendon to the tuberosity, made an incomplete recovery or no recovery at all. Capsulitis-There were sixty-one patients with capsulitis. All were examined under anaesthesia so that they could be differentiated into the sub-groups of irritative and adhesive capsulitis.

Irritative capsulitis-Twenty patients belonged to this group and were first seen after an average period of seven weeks from the onset of symptoms. Pain was severe and almost constant, and it was worse in bed. Movement was entirely scapulo-thoracic and elevation was to $\mathbf{7 0}$ degrees only. Under anaesthesia full movement at the scapulo-humeral joint was possible. Treatment was primarily by rest in an abduction splint or sling, no evidence being found that the splint had any advantage over the sling. Indeed several patients treated in a splint had to discard it because the pain was increased. Gentle exercises were encouraged at an early stage, the exercises first being practised with the patient supine; they were encouraged by heat in its various forms. On the average, full function with a normal range of painless movement was restored within three and a half months, although three patients developed adhesive capsulitis and needed manipulations before recovery was complete.

Adhesive capsulitis-There were forty-one patients in this group who first attended for treatment after an average period of eight months. Pain was less severe, and it was felt only after attempted movement of the shoulder which was mainly scapulo-thoracic. The range of movement was not altered under anaesthesia. When movement was forced, adhesions were felt and heard to tear. In this group the primary treatment was by manipulation under anaesthesia. Since lateral rotation of the humerus is necessary for full abduction, this movement should be carried out first; in most cases this alone was sufficient to free the shoulder. After manipulation the limb was supported in an abduction splint for two weeks and active exercises were practised from the beginning. There was full recovery within a period ranging from two weeks to four months. In five cases three manipulations were necessary; in the other thirty-six cases one manipulation was sufficient. In the follow-up of this group, crepitus was found constantly during passive movements of the shoulder joint but the patient was usually unaware of it and the significance of such crepitation is uncertain. 


\section{SUMMARY}

1. In a series of one hundred personal cases of " painful shoulder " rupture of the musculotendinous cuff was proved by operation in eighteen cases and was presumed in eight further cases, namely, in about one quarter of the series.

2. Of the eighteen cases treated by operation through the transacromial approach, perfect results were obtained only in four. The other results were unsatisfactory. In five, movement was good but there was pain or weakness of the limb. In nine the condition was unaltered or made worse.

3. Conservative treatment of traumatic lesions of the supraspinatus tendon is therefore advocated. The advisability of rest of the shoulder joint in an abduction splint for five or six weeks is stressed. Operative exposure, through a transacromial approach, is recommended only when conservative measures have failed.

4. When there is rupture of the cuff there is evidence that simple longitudinal suture of the gap after freshening of the margins is likely to give better results than attempts to suture the medial end of the rupture to the greater tuberosity. Most ruptures have a longitudinal extension of the initial transverse tear, and direct suture of the medial edge of the gap to the greater tuberosity may lead to shortening of the tendon and interference with its function.

5. Ruptures of the supraspinatus tendon may cause no symptoms, lead to complete loss of function, or cause a painful arc of movement during mid-elevation. The clinical state depends upon whether or not the condition of the ruptured tendon is causing pain, and whether the other short rotator muscles of the shoulder can compensate in tensor and fixator actions for the loss of action of the supraspinatus.

6. Capsulitis is a convenient term by which to describe inflammatory lesions of the capsule and bursae around the shoulder joint. The initial stage of irritative capsulitis may develop to the later stage of adhesive capsulitis. Differentiation is important because treatment in the first stage is by rest, and in the second by manipulation and exercise.

7. The subdeltoid bursa is the "peritoneum " of the spinatus tendons; like the peritoneum it shares the pathology of the organs it protects and is itself seldom the site of primary pathological processes.

8. The management of the "frozen shoulder," whether loss of movement is protective or adhesive, calls for time and patience but the ultimate outlook is good.

\section{REFERENCES}

Armstrong, J. R. (1947): Lancet, 1, 94.

AxEN, O. (1941): Acta Radiologica, 22, 268.

Bosworth, D. M. (1940): Journal of Bone and Joint Surgery, 22, 369.

Burns, B. H., and Ellis, V. H. (1937): Recent Advances in Orthopaedics. London: Churchill, 151.

Codman, E. A., and Akerson, I. B. (1931): Annals of Surgery, 93, 348.

Codman, E. A. (1931): Surgery, Gynecology and Obstetrics, 52, 579.

Grant, J. C. B., and Smith, C. G. (1948): Anatomical Record, 100, 666.

Harrison, T. G. (1948): Personal communication on Dissections of the Shoulder Joint.

Hey Groves, E. W., and Griffiths, S. J. (1932): Medical Annual. Bristol: John Wright \& Sons, Ltd., 483.

Inman, V. T., Saunders, J. B. de C., and Abbott, Le Roy (1944): Journal of Bone and Joint Surgery. $26,1$. McLaughlin, Harrison L. (1944): Journal of Bone and Joint Surgery, 26, 31.

Mercer, W. (1943): Orthopaedic Surgery. 3rd Edition. London: Edward Arnold \& Co., 667.

Neviaser, J. S. (1945): Journal of Bone and Joint Surgery, 27, 211.

Watkins, J. T. (1925): Journal of Bone and Joint Surgery, 7, 787.

Wilson, P. D. (1931): Journal of the American Medical Association, 96, 433.

Withers, R. J. W. (1942): Ulster Medical Journal, 11, 1.

vol. $31 \mathrm{~B}$, No. 3 , AUgust 1949 